\title{
Delayed administration of a matrix metalloproteinase inhibitor limits progressive brain injury after hypoxia-ischemia in the neonatal rat
}

\author{
Christopher C Leonardo ${ }^{1}$, Autumn K Eakin ${ }^{1}$, Joanne M Ajmo1, \\ Lisa A Collier ${ }^{1}$, Keith R Pennypacker ${ }^{1}$, Alex Y Strongin ${ }^{3}$ and \\ Paul E Gottschall*2
}

Address: ${ }^{1}$ Department of Molecular Pharmacology and Physiology, School of Basic Biomedical Sciences, College of Medicine, University of South Florida, Tampa, FL, 33612, USA, ${ }^{2}$ University of Arkansas for Medical Sciences, Department of Pharmacology and Toxicology, Little Rock, AR, 72205, USA and ${ }^{3}$ Burnham Institute for Medical Research, La Jolla, CA, 92037, USA

Email: Christopher C Leonardo - cleonard@health.usf.edu; Autumn K Eakin - aeakin@health.usf.edu;

Joanne M Ajmo - jmayer1@health.usf.edu; Lisa A Collier - lcollier@health.usf.edu; Keith R Pennypacker - kpennypa@health.usf.edu;

Alex Y Strongin - strongin@burnham.org; Paul E Gottschall* - pegottschall@uams.edu

* Corresponding author

Published: II August 2008

Journal of Neuroinflammation 2008, 5:34 doi:10.1186/1742-2094-5-34
Received: 22 May 2008

Accepted: II August 2008

This article is available from: http://www.jneuroinflammation.com/content/5/I/34

(c) 2008 Leonardo et al; licensee BioMed Central Ltd.

This is an Open Access article distributed under the terms of the Creative Commons Attribution License (http://creativecommons.org/licenses/by/2.0), which permits unrestricted use, distribution, and reproduction in any medium, provided the original work is properly cited.

\begin{abstract}
Background: Hypoxia-ischemia $(\mathrm{H}-\mathrm{I})$ can produce widespread neurodegeneration and deep cerebral white matter injury in the neonate. Resident microglia and invading leukocytes promote lesion progression by releasing reactive oxygen species, proteases and other pro-inflammatory mediators. After injury, expression of the gelatin-degrading matrix metalloproteinases (MMPs), MMP-2 and MMP-9, are thought to result in the proteolysis of extracellular matrix (ECM), activation of cytokines/chemokines, and the loss of vascular integrity. Thus, therapies targeting ECM degradation and progressive neuroinflammation may be beneficial in reducing $\mathrm{H}-\mathrm{I}$ - induced neuropathy. Minocycline has MMP-inhibitory properties and is both anti-inflammatory and neuroprotective. AG3340 (prinomastat) is an MMP inhibitor with high selectivity for the gelatinases. The purpose of this study was to determine whether these compounds could limit $\mathrm{H}-\mathrm{I}-$ induced injury when administered at a delayed time point.
\end{abstract}

Methods: Sprague-Dawley rats were exposed to $\mathrm{H}-\mathrm{I}$ at postnatal day 7 (P7), consisting of unilateral carotid artery ligation followed by $90 \mathrm{~min}$ exposure to $8 \% \mathrm{O}_{2}$. Minocycline, AG3340, or vehicle were administered once daily for 6 days, beginning 24 hours after insult. Animals were sacrificed at PI 4 for neurohistological assessments. Immunohistochemistry was performed to determine the degree of reactive astrogliosis and immune cell activation/recruitment. Neural injury was detected using the Fluoro-Jade stain, a marker that identifies degenerating cells.

Results: CDIIb and glial fibrillary acidic protein (GFAP) immunopositive cells increased in ipsilateral cortex after treatment with vehicle alone, demonstrating microglia/macrophage recruitment and reactive astrogliosis, respectively. Fluoro-Jade staining was markedly increased throughout the fronto-parietal cortex, striatum and hippocampus. Treatment with minocycline or AG3340 inhibited microglia/macrophage recruitment, attenuated astrogliosis and reduced Fluoro-Jade staining when compared to vehicle alone.

Conclusion: The selective gelatinase inhibitor AG3340 showed equal efficacy in reducing neural injury and dampening neuroinflammation when compared to the anti-inflammatory compound minocycline. Thus, MMP-2 and MMP-9 may be viable therapeutic targets to treat neonatal brain injury. 


\section{Background}

Exposure to a hypoxic-ischemic (H-I) insult has distinctive consequences in the developing brain. An immature vasculature and low baseline blood flow render the neonatal brain susceptible to even modest changes in perfusion pressure [1]. The maladaptive neurobiological response can be severe, resulting in deep cerebral white matter injury and substantial neuronal loss [2]. Similar to the human condition, neonatal rodent models recapitulating these injuries show cortical and subcortical infarctions, impaired motor function [3-5] and cognitive deficits [6-8]. Previous studies have linked oxidative stress $[9,10]$ and NMDA receptor activation [11] to white matter injury, while glutamatergic blockade has been shown to reduce H-I-induced infarction [12-14] and white matter damage [15]. Though excitotoxicity and free radical production are key contributors to the neuropathology of these lesions, there is a growing interest in identifying additional therapies to limit the progressive neuroinflammation that accompanies ischemic injury.

Inflammation occurs in response to injury and initiates pathological responses that potentiate neural injury. The release of proteases from activated glia results in proteolytic degradation of basement membrane constituents. This breakdown compromises the blood brain barrier, likely allowing entry of peripheral neutrophils and macrophages into the brain. These cells, along with resident microglia, secrete pro-inflammatory cytokines and chemokines that further enhance microglia/macrophage recruitment to the injured site. Matrix metalloproteinases (MMPs), the most well-studied extracellular matrix (ECM)-degrading proteases, are capable of processing TNF- $\alpha$ [16], IL-1 $\beta$ [17] and SDF- $1 \alpha$ [18] to their biologically active forms. Several MMPs, particularly the gelatinases, are elevated after cerebral ischemia and have been shown to degrade basement membrane proteins $[19,20]$. In culture, MMP-2 - positive astrocytes produced MMP-9 when stimulated with either TNF- $\alpha$ or IL-1 $\beta$ [21]. In vivo, MMP-2 expression increased in astrocytic endfeet of rats exposed to MCAO, while MMP-9 expression was localized to neutrophils and endothelial cells [22]. Interestingly, elevated gelatinolytic activity colocalized with neuronal laminin degradation after focal ischemia, effects that were attenuated after administration of a highly selective MMP inhibitor [23]. In agreement with these data, mice lacking MMP-9 showed improved outcomes that were directly related to reduced microglial activation [24], attenuated blood brain barrier degradation [25] and limited white matter damage [26] after H-I.

In addition to cytokines and basement membrane proteins, MMPs cleave ECM chondroitin sulfate proteoglycans (CSPGs). Proteolytic processing of ECM proteoglycans has been linked to $\mathrm{H}-\mathrm{I}$ pathology in the rat neonate [27], and recent data showed altered proteoglycan expression that was associated with progressive injury [28]. While CSPGs are known substrates for several families of matrix-degrading proteases $[13,29,30]$, little is known about the precise regulation of proteoglycan turnover after H-I.

Although neuropathological outcomes show improvement in MMP null mice [24-26], the need for clinically relevant therapeutic intervention remains. In this study, two compounds were selected to determine the effects of MMP inhibition on neuroinflammation and neural injury after H-I. Minocycline, a tetracycline derivative known for its anti-inflammatory properties, is neuroprotective in several rat injury models [31-33] and has recently been shown to inhibit MMP activity both in vitro and in vivo [34]. AG3340, a small molecule hydroxamate-based inhibitor of MMPs, is efficacious in limiting tumor growth in rodent models $[35,36]$ and was shown to be neuroprotective in adult rodents exposed to chronic ischemia [37]. While both compounds demonstrate good oral bioavailability, AG3340 is a potent MMP inhibitor with high nanomolar affinity for gelatinases, specifically, when compared to the broad anti-inflammatory actions of minocycline. Results here show that both minocycline and AG3340 reduced neuroinflammation and neural injury when administered 24 hrs after H-I, highlighting the potential of targeting MMPs when developing therapies to combat neonatal H-I injury.

\section{Methods}

\section{Induction of hypoxia-ischemia}

All animal procedures were conducted in accordance with the NIH Guide for the Care and Use of Laboratory Animals with a protocol approved by the Institutional Animal Care and Use Committee at the University of South Florida. The number of animals used in this study was limited to the fewest number to complete the project. Neonatal Sprague-Dawley rats were birthed from timepregnant dams (Harlan Labs). Dams and litters were maintained on a 12 hour light/dark cycle ( 7 am - 7 pm) and given access to food and water ad libitum. Litters from 2 dams were culled to 10 pups per litter at postnatal day 1 (P1) and cross-fostered randomly between dams prior to each experiment. Two H-I experiments consisting of animals from 2 litters per experiment were used in this study. The H-I procedure was originally developed by Levine [38] and reprised by Vannucci and colleagues $[39,40]$ for use in the neonate. The H-I methodology was described in some detail previously [28]. Briefly, the procedure entailed permanent unilateral ligation of the common carotid artery followed by transient exposure to hypoxia. P7 rats were anesthetized with $2.5 \%$ isofluorane, placed on a heating pad $\left(37^{\circ} \mathrm{C}\right)$ and maintained at $350 \mathrm{ml} / \mathrm{min}$ of oxygen and $1.5 \%$ isofluorane with an interfaced scav- 
enging system for the duration of the surgery. The right common carotid artery was exposed, isolated away from the vagus and ligated using a 6.0 nylon suture. The musculature and skin were sutured, animals placed back with their corresponding dams for a $2 \mathrm{~h}$ recovery period, and subsequently exposed to $8 \%$ oxygen/ $\mathrm{N}_{2}$ balanced for 90 min. During hypoxia, pups were placed into custommade chambers that maintained a temperature of $37^{\circ} \mathrm{C}$ while permitting the water-saturated oxygen mixture to be dispensed at a constant flow rate [27]. Pups were then returned to their respective dams until initiation of the treatment phase.

\section{Drug treatment}

Animals exposed to $\mathrm{H}$-I were randomly assigned to receive vehicle, minocycline (Sigma Aldrich, St. Louis, MO) or AG3340 (AG3340 was kindly provided by Dr. Peter Baciu, Allergan, Irvine, CA). Vehicle consisted of 50\% DMSO + $25 \%$ propylene glycol in distilled water. Minocycline and AG3340 were dissolved in vehicle to obtain stock solutions of $13.5 \mathrm{mg} / \mathrm{ml}$. Fresh stock solutions of drugs were prepared every 2 days. Pups were weighed each day and weight-based injection volumes were calculated to yield a final dose of $45 \mathrm{mg} / \mathrm{kg}$ for each animal. Treatments were administered once daily (s.c.) for 6 days beginning 24 hours after H-I (P8). Animals were evaluated daily for signs of pain or discomfort, and no adverse effects were observed in response to either compound or vehicle alone. At the end of the treatment period, animals were sacrificed for histochemical analyses.

\section{Tissue preparation}

For histochemical evaluation, tissues were collected 7 days after H-I (P14) as previously described [28]. Animals were anesthetized with pentobarbital $(60 \mathrm{mg} / \mathrm{kg})$ and intracardially perfused with phosphate-buffered saline (PBS, $\mathrm{pH} 7.4$ ) followed by $4 \%$ paraformaldehyde. Brains were removed and cryopreserved with increasing sucrose concentrations $(15 \%, 30 \%), 30 \mu \mathrm{m}$ thick sections were cut on a cryostat, and the sections were thaw-mounted onto Superfrost slides (Fisher Scientific, Suwane, GA). Direct mounting onto slides achieved maximum preservation of morphology of the tissue surrounding the lesion. Serial sections were collected throughout the brain beginning at approximately $1.2 \mathrm{~mm}$ rostral to bregma and ending at approximately $5.8 \mathrm{~mm}$ caudal to bregma.

\section{Histology and immunohistochemistry}

Immunohistochemistry was performed as previously described [28] to assess reactive astrogliosis and microglia/macrophage recruitment to the injured site. Slides were rinsed with PBS, permeabilized and blocked for 60 min (3\% Triton-X, 3\% 1 M Lysine, 10\% NGS in PBS), incubated overnight with primary antibody at $4{ }^{\circ} \mathrm{C}$, washed three times with PBS, incubated for 60 min with fluorescent-tagged secondary antibody at room temperature, and coverslipped using Vectashield aqueous mounting media (Vector Labs, Burlingame, CA). Double-label immunohistochemistry was achieved by co-incubation with anti-mouse and anti-rabbit primary antibodies, and subsequent co-incubation with secondary antibodies conjugated to distinct fluorophores for each respective species of primary antibody.

Primary antibodies used in these studies were mouse antiglial fibrillary acidic protein (GFAP) (Roche Applied Science, Indianapolis, IN; $1: 1000)$ and mouse anti-OX-42 (Serotec, Raleigh, NC; 1:3000). Primary antibodies were visualized using either Alexa Fluor 488 (green) or Alexa Fluor 594 (red) secondary antibodies (Molecular Probes, Eugene, OR). Working concentrations for secondary antibodies were 1:300 for OX-42 and 1:1000 for all other primary antibodies.

In tissue sections from the central nervous system, FluoroJade stain mainly identifies areas of neural injury, predominantly degenerating neurons, and provides a positive quantitative marker as opposed to the absence of signal when using Nissl stain. Fluoro-Jade was previously shown to be a more sensitive measure of neural injury when compared to triphenyltetrazolium chloride [41]. This method was adapted from Schmued and colleagues [42] and subsequently detailed [41]. Tissues mounted on glass slides were sequentially placed in $100 \%$ ethanol for $3 \mathrm{~min}, 70 \%$ ethanol for $1 \mathrm{~min}$ and deionized water for 1 min. Sections were oxidized for $15 \mathrm{~min}$ using $0.06 \%$ $\mathrm{KMnO}_{4}$ solution followed by 3 brief rinses in PBS. Slides were then immersed in a $0.001 \%$ solution of Fluoro-Jade (Histochem, Jefferson, AR) in $0.1 \%$ acetic acid for $30 \mathrm{~min}$, rinsed with PBS, dried for $20 \mathrm{~min}$ at $45^{\circ} \mathrm{C}$, cleared with xylene and coverslipped using DPX medium (Electron Microscopy Sciences, Ft. Washington, PA).

\section{Image analyses and quantification}

Images were acquired using a Zeiss Axioscope 2 (model \#801572) controlled by Openlab (Improvision Ltd, Lexington, MA) software, and photomicrographs were captured with a Zeiss Axicam Color (model \#412-312) camera. All images subjected to direct comparisons were captured at the same exposure and digital gain settings to eliminate confounds of differential background intensity or false-positive immunoreactivity across sections. Immunoreactivity was quantified using NIH ImageJ software. Photomicrographs of sections from P14 rats were imported into Image and two distinct methods were employed to best assess relative abundance of immunoreactivity. Histogram analyses were performed to assess astrogliosis. Total GFAP intensity values were summed based upon the frequency of positive pixels that occurred within an intensity spectrum ranging from 0 (no immun- 
ofluorescence detected) to 256 (highest immunofluorescence intensity). For GFAP quantification, 2 cortical fields per section were selected for analyses. Cortical field selection was achieved by moving lateral from the most dorsal aspect of the corpus callosum (layers 1-4), and again moving medial-ventral to the adjacent field (layers 5-6). For neural injury indicated by Fluoro-Jade stain, the entire cortex was traced and background subtraction was achieved by enhancing contrast until background particles were eliminated from the images. All sections were selected at fixed intervals throughout the bregma coordinates indicated in 'tissue preparation'.

\section{Statistical analyses}

Data from all treatment groups were expressed as $\mathrm{X} \pm \mathrm{SEM}$. For reactive astrogliosis and cell death, data were represented as total immunofluorescence intensity. Group means were then subjected to a one-way ANOVA with "p value" set at 0.05 . Pair-wise comparisons of group means were made using a Dunnett's Multiple Comparison test.

\section{Results \\ Microglia/macrophage recruitment is associated with neural injury}

Exposure of the neonate to H-I initiates injurious biochemical cascades that facilitate recruitment of proinflammatory cells to the lesion site. An early and sustained indicator of CNS injury is the activation and recruitment of microglia and macrophages to the injured site. These cell types are important in the initiation and maintenance of the neuroinflammatory response, including the production of MMPs. Immunohistochemistry was performed on sections from untreated and vehicle-treated animals that were exposed to $\mathrm{H}-\mathrm{I}$ ( $\mathrm{N}=5$ per group) to assess the immune cell response using anti-OX-42, an antibody that binds the CD11b antigen that is expressed on cell surfaces of microglia and macrophages. Intense OX-42 immunoreactivity was detected 7 days after H-I (Figure 1). Robust elevations in OX-42 - positive cells occurred in the ipsilateral cerebral cortex (Figure 1B) when compared to the contralateral control region (Figure 1A). Although some labeling was detected in the contralateral cortex, immunoreactivity was faint and diffuse by comparison. In contrast, cells expressing the CD11b antigen were abundant throughout the striatum of both hemispheres (Figure 1C,D). Closer examination revealed that OX-42 - positive cells displayed an amoeboid morphology in the ipsilateral striatum (Figure 1D) and cortex (Figure $1 \mathrm{E}$ ) that is consistent with activated microglia or macrophages, while CD11b - expressing cells in contralateral striatum exhibited a ramified morphology.

Hypoxia-ischemia often produces cortical and subcortical cavitary infarctions over time that are not present up to 4 days after insult. Tissues from vehicle-treated animals ( $\mathrm{N}$
$=5$ ) were collected 7 days after H-I and stained with Fluoro-Jade to determine the degree of neural injury present at this time (Figure 2). Fluoro-Jade staining was prominent in the ipsilateral hemisphere. Signal appeared as columns in lower cortical layers and was diffusely distributed throughout the striatum (Figure 2A). The cortical and striatal injury profile was consistent with elevated OX-42 immunoreactivity (Figure 2C, Figure 1D). FluoroJade also stained hippocampal CA1-CA3 pyramidal neurons intensely (Figure 2B), while other hippocampal fields showed little staining. OX-42 immunoreactivity did not colocalize with Fluoro-Jade in the pyramidal cell layers. However, CD11b - positive cells were localized to the molecular layer of dentate gyrus and other regions seated ventral to CA1 (Figure 2D). In general, Fluoro-Jade staining was present in and adjacent to regions that contained increased numbers of activated microglia/macrophages, indicating that the activation of these cells is associated with neural injury.

\section{Delayed treatment dampens reactive astrogliosis}

Previous data demonstrated a robust astroglial response early after $\mathrm{H}-\mathrm{I}$ in the neonate, indicating that reactive astrocytes are key players in neuroinflammation and respond upstream of severe infarction [28]. To test whether inhibition of MMPs can reduce astrocyte reactivity after H-I, immunohistochemistry was performed 7 days after H-I to assess GFAP upregulation (Figure 3 ) in animals treated with vehicle, minocycline or AG3340 for 6 days after H-I. Low levels of GFAP were detected in contralateral cerebral cortex (Figure 3A,G). In general, GFAP immunoreactivity was prominent in ipsilateral somatosensory cortical layers 5-6 (Figure 3C,F,I) with lesser relative intensity in cortical layers $1-4$ (Figure $3 \mathrm{~B}, \mathrm{E}, \mathrm{H}$ ). Animals treated with vehicle alone showed robust increases in GFAP in layers 1-4 (Figure 3B) and layers 56 (Figure 3C). This is consistent with the astroglial response previously reported at an earlier endpoint [28]. Astrogliosis was diminished after administration of either minocycline or AG3340. While the observed effect of minocycline was most evident in cortical layers 1-4 (Figure 3E), administration of AG3340 diminished astrogliosis in layers 1-4 (Figure $3 \mathrm{H}$ ) and appeared more efficacious in layers 5-6 (Figure 3I) when compared to minocycline (Figure 3F). Quantification was performed by analyzing photomicrographs $(\mathrm{N}=8$ per group, 5 sections per animal) for total GFAP immunoreactivity. Results showed significant reductions in GFAP immunoreactivity in cortical layers 1-4 (Figure 3J) and layers 5-6 (Figure $3 \mathrm{~K}$ ) of animals treated with either minocycline or AG3340 compared to treatment with vehicle alone ( $\mathrm{p}<$ 0.05). Despite a clear trend toward diminished fluorescent signal in sections from animals treated with AG3340 relative to minocycline, efficacy did not differ significantly between treatments. 


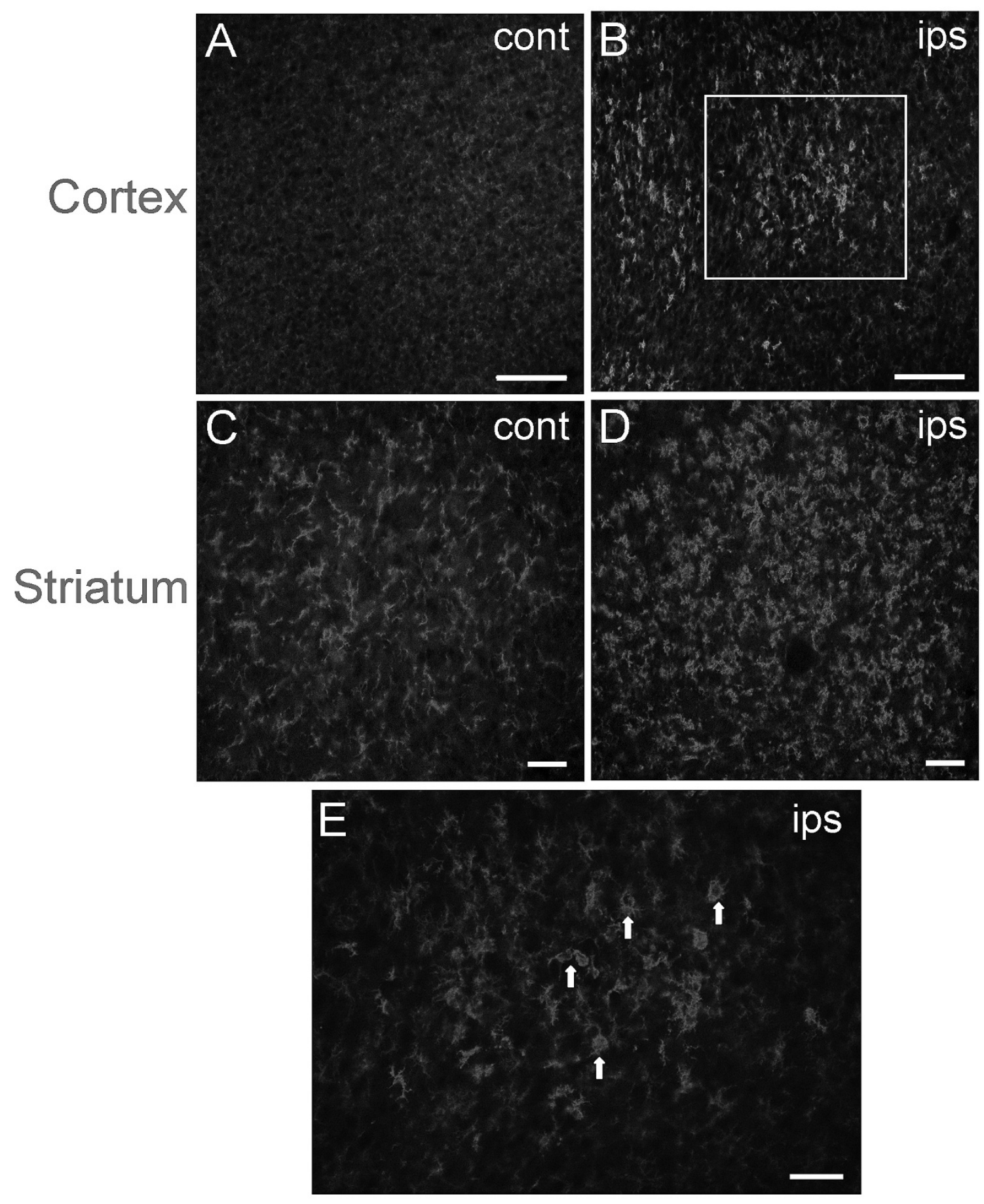

Figure I

Microglia/macrophages are activated and recruited to the lesion site 7 days after $\mathbf{H}-\mathrm{I}$. Exposure to $\mathrm{H}-\mathrm{I}$ at $\mathrm{P7}$ resulted in increased CDI Ib - positive cells, as measured by OX-42 immunoreactivity, in ipsilateral somatosensory cortex (B) compared to contralateral control (A). CDI Ib-expressing cells were abundant in corpus striatum of both hemispheres but showed distinct morphology (C,D). Cells in the ipsilateral striatum displayed the activated amoeboid phenotype (D) similar to those in ipsilateral cortex $(E)$ when compared to the ramified morphology in the contralateral hemisphere (C) that is associated with a less activated state. Scale bars $=100 \mu \mathrm{m}(A, B), 50 \mu \mathrm{m}(C-E)$. 

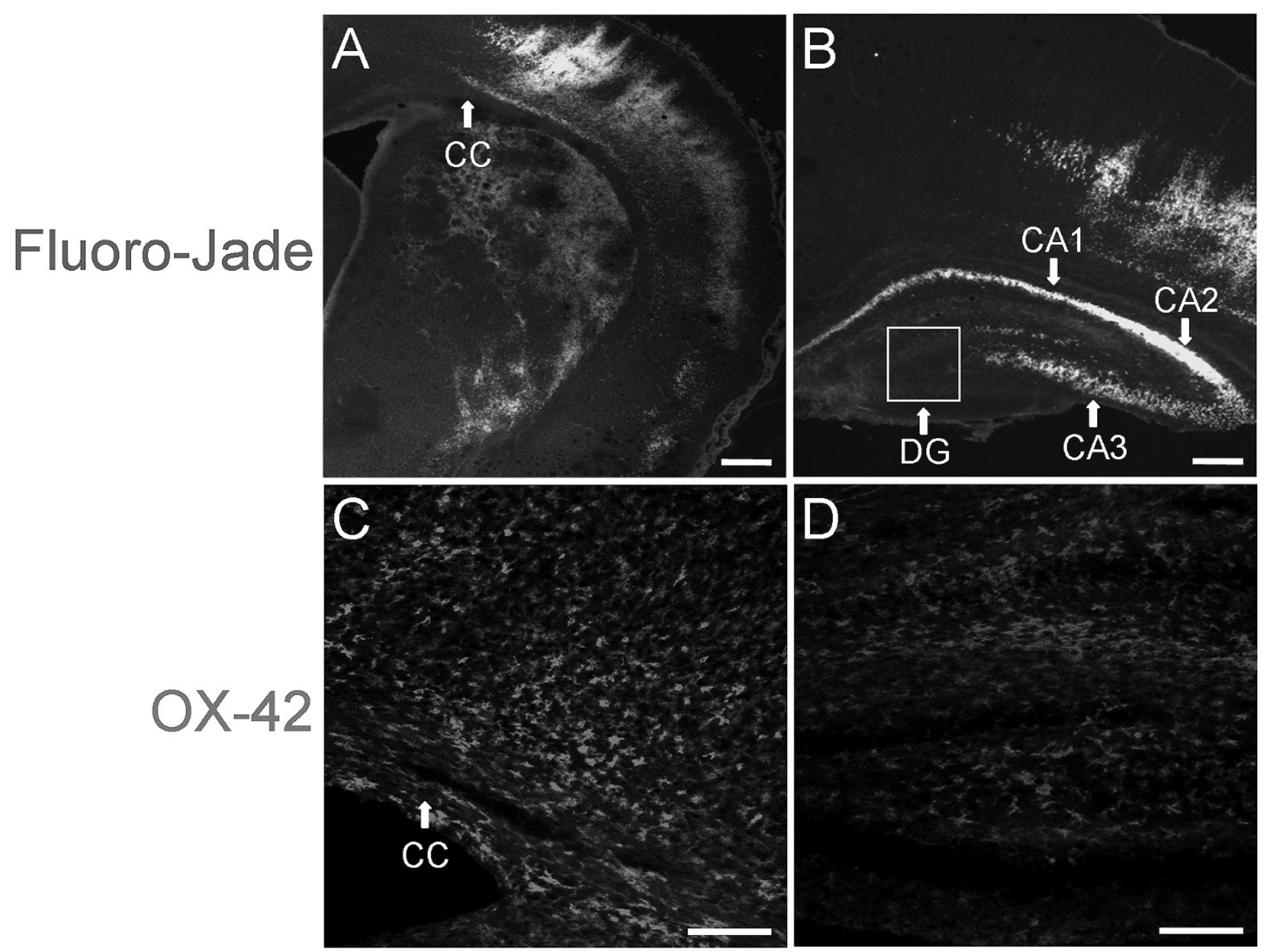

\section{Figure 2}

Microglia/macrophage activation and recruitment are associated with neural injury. Exposure to $H-I$ at $P 7$ resulted in robust Fluoro-Jade staining throughout cerebral cortex, corpus striatum (A) and CAI-CA3 pyramidal cell layers of hippocampus (B), indicating severe neural injury. OX-42 immunoreactivity showed CDI lb-expressing cells at the cortical lesion site (C). Hippocampal OX-42 localized to the molecular layer of dentate gyrus and was evident in hippocampal regions seated ventral to CAI, but was not present in the pyramidal cell layers (D). Scale bars $=100 \mu \mathrm{m}$.

\section{Delayed treatment reduces neural injury}

Marked increases in Fluoro-Jade staining were evident in ipsilateral cerebral cortex, corpus striatum and hippocampus of animals exposed to H-I and treated with vehicle alone (Figure 2,4). Staining was absent from the contralateral hemisphere and very little non-specific background staining was detected (Figure 4A,C,E). Intense columnarshaped immunofluorescence was evident throughout the cerebral cortex in sections that ranged from the initial emergence of lateral ventricles to ventral hippocampus. Treatment with minocycline abolished this effect, showing little or no cortical Fluoro-Jade labeling (Figure 4D). Administration of AG3340 also reduced Fluoro-Jade staining in cerebral cortex (Figure 4F) when compared to sections from animals treated with vehicle alone, though this compound appeared to be less efficacious than minocycline. Quantification of cortical Fluoro-Jade staining was performed by analyzing photomicrographs $(\mathrm{N}=5$ per group, 5 sections per animal) for total area occupied by stain. Results showed a significant reduction in FluoroJade staining after treatment with both compounds (Figure $4 \mathrm{G}$ ) when compared to treatment with vehicle alone $(\mathrm{p}<0.05)$. Efficacy did not differ significantly between minocycline and AG3340. 

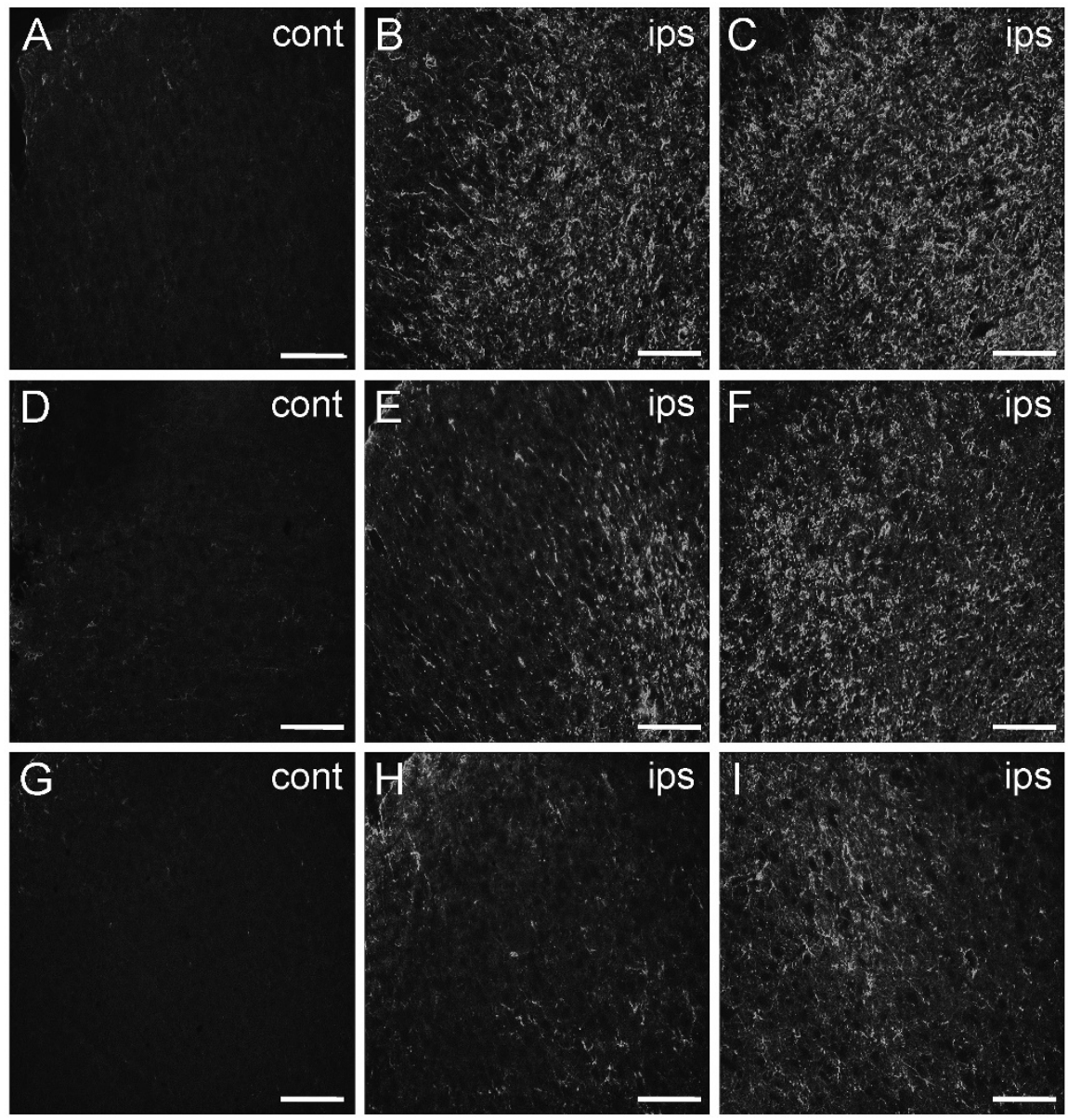
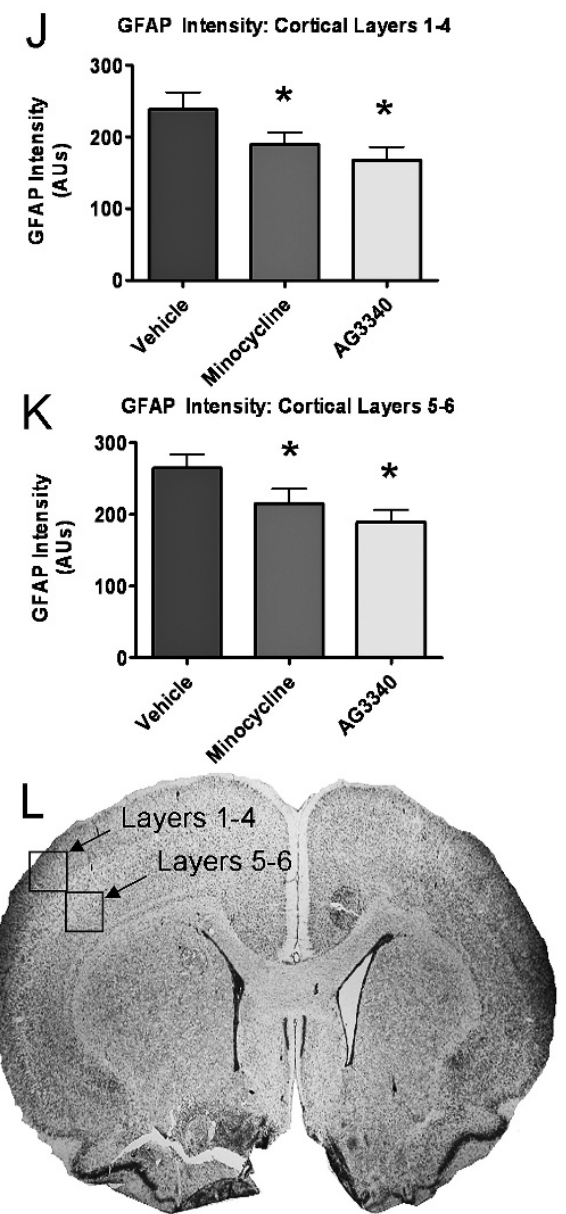

Figure 3

Delayed treatment with minocycline or AG3340 dampens reactive astrogliosis. P7 rat pups were exposed to $\mathrm{H}-\mathrm{I}$ and treated with either vehicle, minocycline or AG3340 once daily for 6 days, beginning 24 hours after insult. (A-C) Astrogliosis, as measured by GFAP immunoreactivity, was markedly elevated in cortical layers I-4 (B) and layers 5-6 (C) compared to contralateral control (A) after treatment with vehicle alone. Administration of either minocycline (D-F) or AG3340 (G-I) 24 hours after $\mathrm{H}-\mathrm{I}$ reduced GFAP immunoreactivity in layers $\mathrm{I}-4(\mathrm{E}, \mathrm{H})$ and layers 5-6 (F,I) compared to control hemisphere $(D, G)$. GFAP immunoreactivity was significantly reduced in cortical layers I-4 (J) and layers 5-6 $(K)$ of animals treated with either minocycline or AG3340 compared to treatment with vehicle alone. $N=8, *=p<0.05$. Scale bars $=100 \mu \mathrm{m}$.

\section{Delayed treatment ameliorates microglia/macrophage response}

To determine whether reduced cell death was associated with microglia/macrophage recruitment to the lesion site, immunohistochemistry was performed with anti-OX-42 to detect the CD11b antigen present on cell membranes of microglia and macrophages. Tissues from animals treated with vehicle alone showed increased OX-42 immunoreactivity in the ipsilateral cerebral cortex (Figure 5B). CD11b labeling in contralateral control regions was faint and diffuse by comparison (Figure 5A), indicating that OX-42 positive cells were recruited to the injured area or that those present markedly increased the expression of CD11b. Treatment with minocycline ameliorated this effect, as ipsilateral OX-42 immunoreactivity (Figure 5D) was indistinguishable from that observed in the contralateral control hemisphere (Figure 5C). OX-42 - positive cells were also greatly reduced in ipsilateral cortex of animals treated with AG3340 (Figure 5F) relative to contralateral control (Figure 5E).

\section{Discussion}

The central nervous system response to hypoxia-ischemia or ischemia reperfusion injury often includes activation of astrocytes, resident microglia, and the infiltration of peripheral leukocytes. Subsequent release of pro-inflammatory cytokines and other inflammatory effectors contribute to neural injury of white and gray matter after H-I 

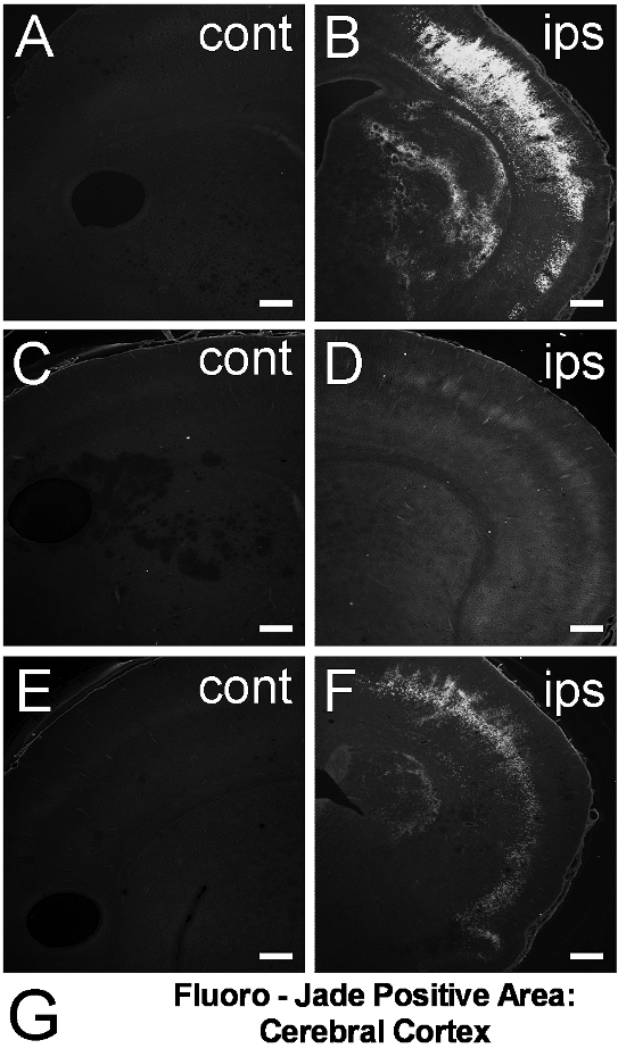

Fluoro - Jade Positive Area Cerebral Cortex

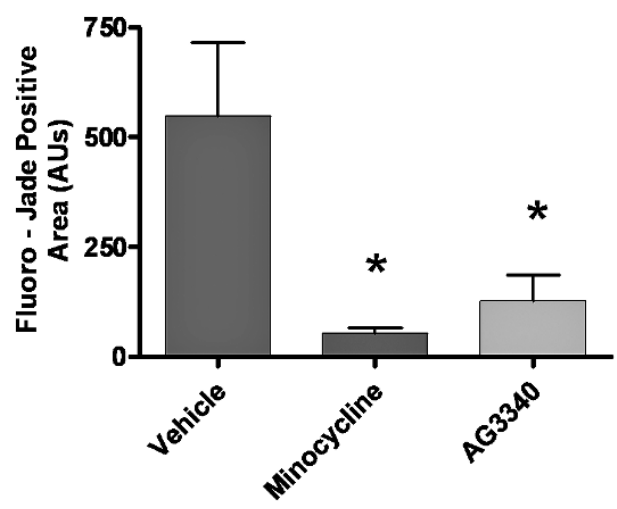

\footnotetext{
Figure 4

Delayed treatment with minocycline or AG3340 reduces neural injury. P7 rat pups were exposed to $\mathrm{H}-\mathrm{I}$ and treated with either vehicle, minocycline or AG3340 once daily for 6 days, beginning 24 hours after insult. Animals treated with vehicle alone showed robust Fluoro-Jade labeling in ipsilateral cortex and corpus striatum (B). Fluoro-jade staining was nearly abolished after treatment with minocycline (D) and greatly reduced after treatment with AG3340 (F) relative to vehicle alone $(B)$. No labeling was detected in contralateral control regions (A,C,E). Quantification showed a significant reduction after treatment with either minocycline or AG3340 (G) compared to treatment with vehicle alone. $\mathrm{N}=5, *=\mathrm{p}<0.05$. Scale bars $=100 \mu \mathrm{m}$.
}

[43]. The present study evaluated the degree of reactive astrogliosis, microglia/macrophage recruitment and neural injury after H-I in the P7 Sprague-Dawley rat neonate. A single daily administration of minocycline or AG3340 for 6 days, beginning 24 hrs after H-I, provided protection against neural injury and dampened astrocyte and microglial activation induced by the insult. Because of the progressive nature of the insult that follows H-I, it is likely that inhibition of inflammatory effectors by minocycline or AG3340 was associated with reduced neural injury.

The neurobiological reaction to H-I-induced lesion involves a progressive inflammatory response that exacerbates the injury via the release of cytokines, chemokines and other effector molecules from reactive astrocytes, resident immune cells and peripheral leukocytes. Robust elevations in GFAP immunoreactivity were prominent throughout the ipsilateral cerebral cortex of animals treated with vehicle alone. Fluoro-Jade histochemistry revealed marked neural injury that was anatomically consistent with heightened GFAP and OX-42 labeling. CD11b - positive cells were abundantly distributed throughout the cortex, striatum and hippocampus after insult. Evidence indicates that resident microglia, and not infiltrating leukocytes, are the primary immune cells that respond to ischemic injury in the neonatal rat brain [44]. Although the majority of $\mathrm{CD} 11 \mathrm{~b}$ - expressing cells detected here displayed an amoeboid morphology characteristic of activated microglia, it is also possible that these cells were peripheral monocytes that had entered the brain after insult.

MMPs, derived mainly from microglia, influence the neurological outcome after hypoxia and/or ischemia-induced lesion in a complex manner. After experimental stroke, MMP-9 null mice showed reduced blood brain barrier degradation and white matter injury [26]. However, more recent data demonstrated that acute expression of MMPs contributes to injury, yet more chronically, MMPs promote plasticity and recovery [45]. Most relevant, the extent of neural injury induced by H-I in the rat neonate was diminished in MMP-9 null mice compared to the lesion in wild type mice [24]. MMP-9 co-localizes with activated resident microglia [24] and infiltrating leukocytes $[25,46]$ in rodents subjected to cerebral ischemia, indicating a link between activated immune cells and MMP expression. Consistent with these data, the absence of immune cell recruitment after treatment with inhibitors in the present study provides further evidence that MMP activity is linked to neuroinflammation.

Several different mechanisms could account for the neuroprotection afforded by AG3340. MMPs participate in complex injury responses through interactions with and activation of cytokines, chemokines and other pericellular 

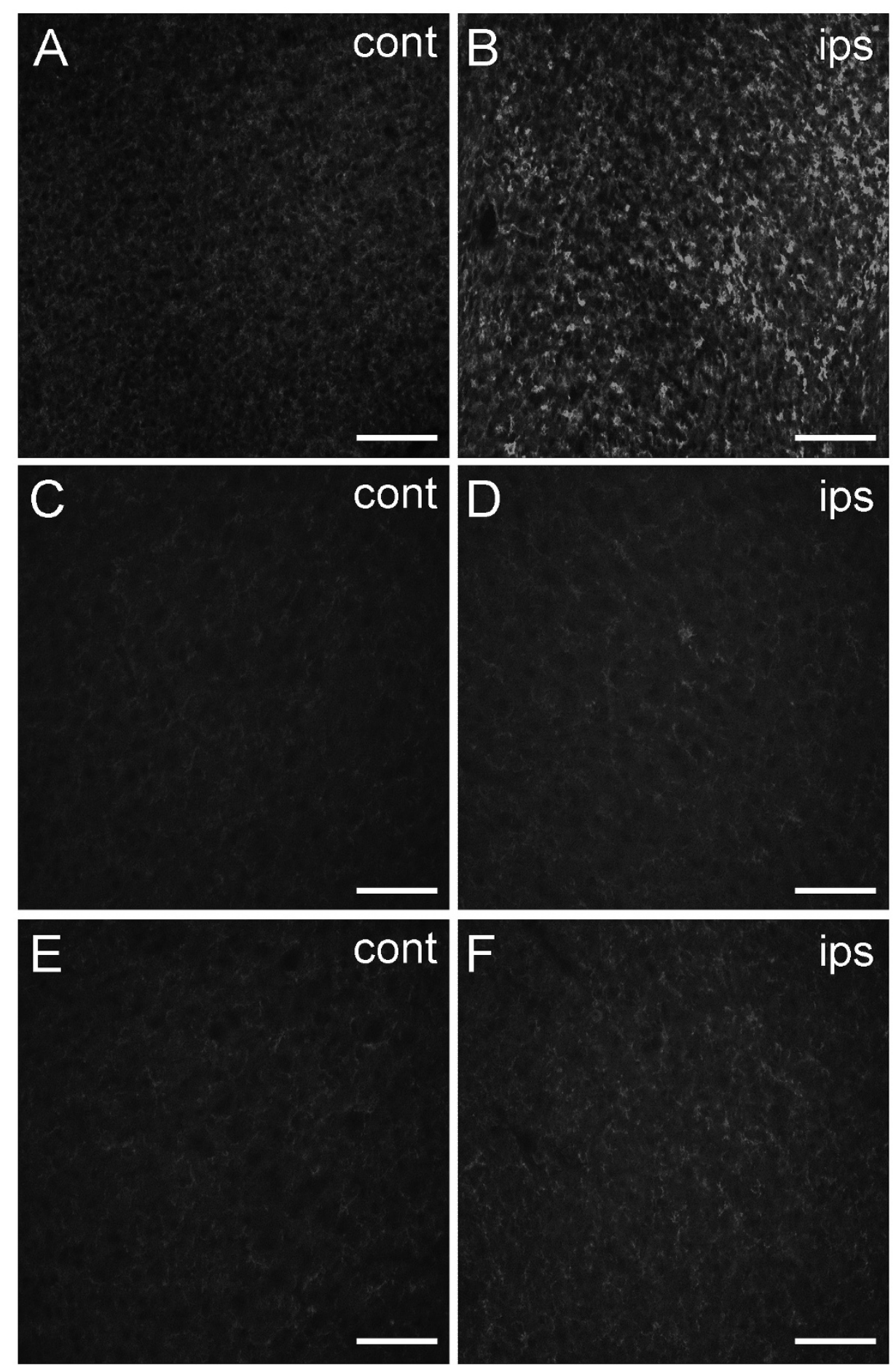

\section{Figure 5}

Delayed treatment with minocycline or AG3340 ameliorates the microglia/macrophage response. P7 rat pups were exposed to $\mathrm{H}-\mathrm{I}$ and treated with either vehicle, minocycline or AG3340 once daily for 6 days, beginning 24 hours after insult. Intense OX-42 immunoreactivity was evident throughout the ipsilateral cerebral cortex of animals treated with vehicle alone (B). Microglia/macrophage labeling was nearly abolished after treatment with minocycline (D) and was markedly reduced after treatment with AG3340 (F). OX-42 immunoreactivity in contralateral control regions was faint and diffuse by comparison (A,C,E). Scale bars $=100 \mu \mathrm{m}$. 
and cell surface substrates [47], and inhibition would limit the action of these pro-inflammatory agents $[16,45]$. Because gelatinases efficiently degrade basement membrane proteins, it is possible that the actions of the MMP inhibitor resulted in preservation of basement membrane proteins and an intact blood brain barrier. Another possible mechanism of the MMP inhibitor could be that a loss of ECM proteolysis derived from microglia limited their ability to migrate through the ECM to the region of injury (or other unknown mechanisms). This is an interesting potential mechanism which, to date, has not been investigated. Data from the present study indirectly support such a mechanism since microglia/macrophage density in the injured cerebral cortex was diminished in animals treated with AG3340. Additionally, results here showed that delayed administration of AG3340 $24 \mathrm{~h}$ after H-I limited microglial numbers and conferred protection from neural injury. This suggests that selective targeting of MMPs even after an episode of H-I may be a clinically relevant therapeutic approach.

Minocycline is known for its broad anti-inflammatory actions and has recently been shown to inhibit MMP activity in an in vivo stroke model [34]. In the present study, we sought to determine the degree to which two compounds that exhibit distinct anti-inflammatory actions and affinities for MMPs, differentially influence neural injury caused by $\mathrm{H}-\mathrm{I}$ in the neonate. In a manner similar to AG3340, minocycline reduced microglia/macrophage recruitment and cortical injury after H-I. Both compounds also inhibited astroglial reactivity in the injured region, suggesting that MMPs may contribute to the activation of astrocytes as well. Two other neonatal $\mathrm{H}$ I studies have demonstrated short-term [31] and longterm [32] protection after treatment with minocycline. Importantly, these experiments differed in that animals were pretreated prior to insult and again immediately after occlusion, whereas in the present study, treatment did not begin until 24 hours after the insult.

\section{Conclusion}

The present study indicates that treatment with either AG3340, a compound that is relatively selective for gelatin-degrading MMPs, or minocycline, a tetracycline derivative with anti-inflammatory and MMP-inhibitory properties, protected the neonatal brain even when first administered $24 \mathrm{~h}$ after $\mathrm{H}-\mathrm{I}$, a clinically relevant time point. Future investigations are necessary to define the early time course for efficacy, the specific pro-inflammatory mechanisms involved in progressive $\mathrm{H}-\mathrm{I}$ injury, and the degree to which the neuroprotective effects of these compounds are dependent on MMP activity. These studies, in conjunction with data reported here, will aid not only in identifying the specific sequence of neuroinflammatory events that contribute to H-I injury, but also in determining whether MMPs mediate these processes and therefore represent attractive therapeutic targets.

\section{Competing interests}

The authors declare that they have no competing interests.

\section{Authors' contributions}

CCL and PEG designed the experiments. CCL performed the H-I procedure, drug injections, brain sectioning, immunohistochemistry, data analysis, and wrote the manuscript. AKE assisted with sectioning and immunohistochemistry. LAC performed the Fluoro-Jade histochemistry. PEG directed this work, contributing to the data analysis and writing of the manuscript. All authors edited and approved the final manuscript.

\section{Acknowledgements}

This work was supported in part by Shriners Research Grant 8560 and American Heart Association Grant-in-Aid 05552 I6B (P.E.G.). AG3340 was kindly provided by Dr. Peter Baciu.

\section{References}

I. Baier RJ: Genetics of perinatal brain injury in the preterm infant. Front Biosci 2006, I I: I37|- I387.

2. Back SA, Luo NL, Borenstein NS, Levine JM, Volpe JJ, Kinney HC: Late oligodendrocyte progenitors coincide with the developmental window of vulnerability for human perinatal white matter injury. J Neurosci 200I, 2 I (4): | 302- I3 I 2.

3. Bona E, Johansson BB, Hagberg $H$ : Sensorimotor function and neuropathology five to six weeks after hypoxia-ischemia in seven-day-old rats. Pediatr Res 1997, 42(5):678-683.

4. Jansen EM, Low WC: Long-term effects of neonatal ischemichypoxic brain injury on sensorimotor and locomotor tasks in rats. Behav Brain Res 1996, 78(2): 189-194.

5. Ten VS, Bradley-Moore M, Gingrich JA, Stark RI, Pinsky DJ: Brain injury and neurofunctional deficit in neonatal mice with hypoxic-ischemic encephalopathy. Behav Brain Res 2003, I45( I2):209-219.

6. Young RS, Kolonich J, Woods CL, Yagel SK: Behavioral performance of rats following neonatal hypoxia-ischemia. Stroke I986, I7(6): $1313-1316$.

7. Arteni NS, Salgueiro J, Torres I, Achaval M, Netto CA: Neonatal cerebral hypoxia-ischemia causes lateralized memory impairments in the adult rat. Brain Res 2003, 973(2): I7I-I78.

8. Ikeda T, Mishima K, Yoshikawa T, Iwasaki K, Fujiwara M, Xia YX, Ikenoue T: Selective and long-term learning impairment following neonatal hypoxic-ischemic brain insult in rats. Behav Brain Res 200I, I I 8(I): I7-25.

9. Back SA, Gan X, Li Y, Rosenberg PA, Volpe JJ: Maturation-dependent vulnerability of oligodendrocytes to oxidative stressinduced death caused by glutathione depletion. I Neurosci 1998, I 8(1 6):624I-6253.

10. Bernardo A, Greco A, Levi G, Minghetti L: Differential lipid peroxidation, Mn superoxide, and bcl-2 expression contribute to the maturation-dependent vulnerability of oligodendrocytes to oxidative stress. J Neuropathol Exp Neurol 2003, 62(5):509-5I9.

II. Kaur C, Sivakumar V, Ang LS, Sundaresan A: Hypoxic damage to the periventricular white matter in neonatal brain: role of vascular endothelial growth factor, nitric oxide and excitotoxicity. J Neurochem 2006, 98(4): I200-I2I6.

12. David HN, Leveille F, Chazalviel L, MacKenzie ET, Buisson A, Lemaire $M$, Abraini JH: Reduction of ischemic brain damage by nitrous oxide and xenon. I Cereb Blood Flow Metab 2003, 23(10): I I 68- I 173.

13. Dingley J, Tooley J, Porter H, Thoresen $\mathrm{M}$ : Xenon provides shortterm neuroprotection in neonatal rats when administered after hypoxia-ischemia. Stroke 2006, 37(2):501-506.

14. Puka-Sundvall M, Hallin U, Zhu C, Wang X, Karlsson JO, Blomgren K, Hagberg $H$ : NMDA blockade attenuates caspase-3 activation 
and DNA fragmentation after neonatal hypoxia-ischemia. Neuroreport 2000, I I(I3):2833-2836.

15. Follett PL, Rosenberg PA, Volpe JJ, Jensen FE: NBQX attenuates excitotoxic injury in developing white matter. J Neurosci 2000 , 20(24):9235-924I.

16. Gearing AJ, Beckett P, Christodoulou M, Churchill M, Clements J, Davidson AH, Drummond AH, Galloway WA, Gilbert R, Gordon JL, et al: Processing of tumour necrosis factor-alpha precursor by metalloproteinases. Nature 1994, 370(6490):555-557.

17. Schonbeck U, Mach F, Libby P: Generation of biologically active IL-I beta by matrix metalloproteinases: a novel caspase-Iindependent pathway of IL-I beta processing. J Immunol I998, I 6 I (7):3340-3346.

18. Overall CM, McQuibban GA, Clark-Lewis I: Discovery of chemokine substrates for matrix metalloproteinases by exosite scanning: a new tool for degradomics. Biol Chem 2002, 383(78): $1059-1066$.

19. Cunningham LA, Wetzel M, Rosenberg GA: Multiple roles for MMPs and TIMPs in cerebral ischemia. Glia 2005, 50(4):329-339.

20. Manicone AM, McGuire JK: Matrix metalloproteinases as modulators of inflammation. Semin Cell Dev Biol 2007.

21. Gottschall PE, Yu X: Cytokines regulate gelatinase $A$ and $B$ (matrix metalloproteinase 2 and 9) activity in cultured rat astrocytes. J Neurochem 1995, 64(4): 1513-1520.

22. Rosenberg GA, Cunningham LA, Wallace J, Alexander S, Estrada EY, Grossetete M, Razhagi A, Miller K, Gearing A: Immunohistochemistry of matrix metalloproteinases in reperfusion injury to rat brain: activation of MMP-9 linked to stromelysin- $I$ and microglia in cell cultures. Brain Res 200I, 893(I-2): I04-I I 2.

23. Gu Z, Cui J, Brown S, Fridman R, Mobashery S, Strongin AY, Lipton SA: A highly specific inhibitor of matrix metalloproteinase-9 rescues laminin from proteolysis and neurons from apoptosis in transient focal cerebral ischemia. I Neurosci 2005, 25(27):640I-6408.

24. Svedin P, Hagberg H, Savman K, Zhu C, Mallard C: Matrix metalloproteinase-9 gene knock-out protects the immature brain after cerebral hypoxia-ischemia. I Neurosci 2007, 27(7): $15|1-15| 8$.

25. Gidday JM, Gasche YG, Copin JC, Shah AR, Perez RS, Shapiro SD, Chan PH, Park TS: Leukocyte-derived matrix metalloproteinase-9 mediates blood-brain barrier breakdown and is proinflammatory after transient focal cerebral ischemia. Am J Physiol Heart Circ Physiol 2005, 289(2):H558-68.

26. Asahi M, Wang X, Mori T, Sumii T, Jung JC, Moskowitz MA, Fini ME, Lo EH: Effects of matrix metalloproteinase-9 gene knock-out on the proteolysis of blood-brain barrier and white matter components after cerebral ischemia. I Neurosci 200I, 2I(19):7724-7732.

27. Aya-ay J, Mayer J, Eakin AK, Muffly BG, Anello M, Sandy JD, Gottschall PE: The effect of hypoxic-ischemic brain injury in perinatal rats on the abundance and proteolysis of brevican and NG2. Exp Neurol 2005, I 93(I): 149-162.

28. Leonardo CC, Eakin AK, Ajmo JM, Gottschall PE: Versican and brevican are expressed with distinct pathology in neonatal hypoxic-ischemic injury. J Neurosci Res 2008, 86(5): I I06- I I I 4.

29. Apte SS: A disintegrin-like and metalloprotease (reprolysin type) with thrombospondin type I motifs: the ADAMTS family. Int J Biochem Cell Biol 2004, 36(6):98I-985.

30. Gottschall PE Sandy, J.D., Zimmermann, D.R.: Substrates for Metalloendopeptidases in the Central Nervous System. In Matrix Metalloproteinases in the Central Nervous System Edited by: Conant KGPE. London, Imperial College Press; 2005:87-II8.

31. Cai Z, Lin S, Fan LW, Pang Y, Rhodes PG: Minocycline alleviates hypoxic-ischemic injury to developing oligodendrocytes in the neonatal rat brain. Neuroscience 2006, 137(2):425-435.

32. Fan LW, Lin S, Pang Y, Rhodes PG, Cai Z: Minocycline attenuates hypoxia-ischemia-induced neurological dysfunction and brain injury in the juvenile rat. Eur J Neurosci 2006, 24(2):34|-350.

33. Wasserman JK, Schlichter LC: Minocycline protects the bloodbrain barrier and reduces edema following intracerebral hemorrhage in the rat. Exp Neurol 2007.

34. Machado LS, Kozak A, Ergul A, Hess DC, Borlongan CV, Fagan SC: Delayed minocycline inhibits ischemia-activated matrix metalloproteinases $\mathbf{2}$ and $\mathbf{9}$ after experimental stroke. BMC Neurosci 2006, 7:56.

35. Alves F, Borchers U, Padge B, Augustin H, Nebendahl K, Kloppel G, Tietze LF: Inhibitory effect of a matrix metalloproteinase inhibitor on growth and spread of human pancreatic ductal adenocarcinoma evaluated in an orthotopic severe combined immunodeficient (SCID) mouse model. Cancer Lett 200I, 165(2):161-170.

36. Price A, Shi Q, Morris D, Wilcox ME, Brasher PM, Rewcastle NB, Shalinsky D, Zou H, Appelt K, Johnston RN, Yong VW, Edwards D, Forsyth $\mathrm{P}$ : Marked inhibition of tumor growth in a malignant glioma tumor model by a novel synthetic matrix metalloproteinase inhibitor AG3340. Clin Cancer Res 1999, 5(4):845-854.

37. Nakaji K, Ihara M, Takahashi C, Itohara S, Noda M, Takahashi R, Tomimoto $\mathrm{H}$ : Matrix metalloproteinase-2 plays a critical role in the pathogenesis of white matter lesions after chronic cerebral hypoperfusion in rodents. Stroke 2006, 37(II):2816-2823.

38. Levine S: Anoxic-ischemic encephalopathy in rats. Am J Pathol 1960, 36:1-17.

39. Rice JE 3rd, Vannucci RC, Brierley JB: The influence of immaturity on hypoxic-ischemic brain damage in the rat. Ann Neurol I98I, 9(2): $|3|-|4|$

40. Vannucci RC, Connor JR, Mauger DT, Palmer C, Smith MB, Towfighi J, Vannucci SJ: Rat model of perinatal hypoxic-ischemic brain damage. J Neurosci Res 1999, 55(2): 158-163.

4I. Duckworth EA, Butler TL, De Mesquita D, Collier SN, Collier L, Pennypacker KR: Temporary focal ischemia in the mouse: technical aspects and patterns of Fluoro-Jade evident neurodegeneration. Brain Res 2005, 1042(I):29-36.

42. Schmued LC, Albertson C, Slikker W Jr.: Fluoro-Jade: a novel fluorochrome for the sensitive and reliable histochemical localization of neuronal degeneration. Brain Res 1997, 75 I (I):37-46.

43. Lo EH, Dalkara T, Moskowitz MA: Mechanisms, challenges and opportunities in stroke. Nat Rev Neurosci 2003, 4(5):399-4I5.

44. Denker SP, Ji S, Dingman A, Lee SY, Derugin N, Wendland MF, Vexler ZS: Macrophages are comprised of resident brain microglia not infiltrating peripheral monocytes acutely after neonatal stroke. J Neurochem 2007, 100(4):893-904.

45. Zhao BQ, Wang S, Kim HY, Storrie H, Rosen BR, Mooney DJ, Wang $X$, Lo EH: Role of matrix metalloproteinases in delayed cortical responses after stroke. Nat Med 2006, I 2(4):44I-445.

46. Romanic AM, White RF, Arleth AJ, Ohlstein EH, Barone FC: Matrix metalloproteinase expression increases after cerebral focal ischemia in rats: inhibition of matrix metalloproteinase-9 reduces infarct size. Stroke 1998, 29(5): 1020-1030.

47. Van Lint P, Libert C: Chemokine and cytokine processing by matrix metalloproteinases and its effect on leukocyte migration and inflammation. J Leukoc Biol 2007, 82(6): |375- I38I.

Publish with Bio Med Central and every scientist can read your work free of charge

"BioMed Central will be the most significant development for disseminating the results of biomedical research in our lifetime."

Sir Paul Nurse, Cancer Research UK

Your research papers will be:

- available free of charge to the entire biomedical community

- peer reviewed and published immediately upon acceptance

- cited in PubMed and archived on PubMed Central

- yours - you keep the copyright
BioMedcentral 